\title{
18 Odins Schatten auf der Durchreise in Rouen
}

Résumé : Dans la partie de sa Chronique des Ducs de Normandie traitant de Rollon, Benoît décrit le passage à Rouen d'un mystérieux cavalier vêtu de gris et qui se déplace sur la Seine sans que son cheval en touche l'eau. Refusant de révéler son identité, il prédit pour la dynastie ducale un avenir de neuf règnes prospères. Sans attendre une rencontre avec Rollon devenu chrétien, il continue, en étapes journalières surhumaines, une chevauchée qui semble sans commencement ni fin. En latin, cette histoire est racontée dès avant 1120 comme étant de notoriété générale. Qui est l'inconnu? En ancien norrois, dans les nombreux récits comparables jusque dans les détails, c'est toujours Odin en personne. Pour la Normandie, il faut conclure que si ce n'est plus lui explicitement, c'est quand même son ombre qui hante le domaine flou, dans l'imagination populaire, entre christianisme et paganisme - domaine dont a su se servir, comme on le voit, même une propagande producale, donc, en principe, chrétienne.

In seiner Chronique des Ducs de Normandie, an der er von etwa 1174 bis spätestens 1189 arbeitete, erzählt uns Benoît eine merkwürdige Episode aus der Zeit Rollos, des ersten Herzogs. Da sie in ihrem narrativen Teil fast zweihundert Verse umfaßt, zu denen noch einhundert Verse ihrer historischen Würdigung durch Benoît kommen, können wir sie hier nur gedrängt paraphrasieren. ${ }^{1}$

Eines Tages erleben die Bürger von Rouen, wie ein stattlicher Reiter in einem Mantel aus kostbarem grauem Stoff ${ }^{2}$ auf dem Wasser der Seine flußaufwärts in die Stadt einreitet, ohne daß auch nur die Füße seines Pferdes naß werden. Über seine Person verweigert er jede Auskunft - außer daß er am Morgen aufgebrochen sei von Rennes, in Avranches (nach $80 \mathrm{~km}$ !) zur dritten Tagesstunde eine Mahlzeit eingenommen habe und nun gegen Abend (nach weiteren $220 \mathrm{~km}$ !) in bequemem Paßgang in Rouen eintreffe. In Avranches habe er übrigens sein Speisemesser vergessen; wer seine Angaben nachprüfen wolle, könne es dort abholen. Ein reicher Bürger lädt den Fremden zum Nachtquartier

1 Fahlin (ed., 1951-54), vv. 9839-10020 bzw. 10122. Der Text fehlt in Hs. T, aber nur, weil dort eine ganze Lage verlorengegangen ist. - Unmittelbar vorher berichtet Benoît Ereignisse von 922-923; doch besagt das für eine genauere zeitliche Einordnung wenig, da er unsere Episode einem Anhang seiner Hauptquelle entnahm und sie deshalb innerhalb von Rollos Herzogszeit irgendwo einordnen mußte (s. Anm. 6).

2 V. 9868 «d'escarlate gris». Escarlate wird im Glossar von Södergård (1967 s. v.) zu Recht mit «drap fin〉 übersetzt. Altfrz. escarlate (und mhd. scharlât, scharlach) bezeichnet recht häufig noch feine Stoffe, die ausdrücklich als nicht-rot, sondern z. B. als lila, weiß, schwarz, braun, grünblau und hier eben als grau bezeichnet werden.

Anmerkung: Erstmals veröffentlicht in: Romanische Forschungen 116 (2004), 214-222.

Ә Open Access. (C 2019 Gustav Adolf Beckmann, publiziert von De Gruyter. (c))BY-NC-ND Dieses Werk ist lizenziert unter der Creative Commons Attribution-NonCommercial-NoDerivatives 4.0 Lizenz.

https://doi.org/10.1515/9783110615692-018 
ein. Andere melden dem Herzog von dem Ereignis. Dieser läßt dem Fremden gebieten, sich ihm vor dem Weiterritt vorzustellen. Der Fremde antwortet, er werde am Morgen bis zur Prime für den Herzog bereitstehen, ${ }^{3}$ reitet dann aber schon bei Tagesanbruch weiter. Der erzürnte Herzog äußert den Verdacht, das sei ein böser Geist gewesen; doch seine Leute, auf die der Fremde einen sehr positiven Eindruck gemacht hat, antworten ihm, der Fremde rechne wohl als Prime einen früheren Zeitpunkt als der Herzog und sei deshalb nicht wortbrüchig geworden. Der Gastgeber des Fremden erzählt, er habe diesen nach der Abendmahlzeit in einem langen Gespräch am Feuer nach der Zukunft der Herzogsdynastie gefragt und die Antwort erhalten, von Rollo an würden neun Herzöge kraftvoll regieren. Nach dem zehnten befragt, habe der Fremde nur nachdenklich die Furchen, die er mit einem Stock in die Asche gezeichnet hatte, wieder eingeebnet. Er habe dem Gastgeber übrigens die Scheide zu dem in Avranches vergessenen Messer geschenkt. Der Herzog sendet nach Avranches, und siehe da, der Bote kommt mit dem Messer zurück.

Benoît schließt nun die Betrachtung an, daß sich die Prophezeiung voll bewahrheitet habe: nach dem neunten Herzog, König Heinrich I., habe sich der zehnte (nämlich Benoîts Gönner König Heinrich II.) erst gegen ungeheure Widerstände schließlich glorreich durchgesetzt. In der Tat: Da Heinrich I. 1135 bei seinem Tod keine legitimen Söhne, wohl aber durch seine Tochter Mathilde seinen damals zweijährigen Enkel Heinrich II. hinterließ, war dieser in Heinrichs I. wie in Benoîts Denken der legitime Erbe; daß bis zu seiner Großjährigkeit ein Zwischenherzog Gottfried von Anjou und ein Zwischenkönig Stephan von Blois auftreten sollten, ist in der Perspektive «Rolloniden als Normannenherzöge` irrelevant. ${ }^{4}$

Als 1883 Hugo Andresen erstmalig Benoîts Chronik quellenkritisch untersuchte, ging er auf die Gestalt des ‘geheimnisvollen Fremden` nicht ein, konnte aber die lat. Quelle der Episode aufzeigen: ${ }^{5}$ die sog. Additamenta ad Historiam Normannorum, Zusätze zu den - wie man heute sagt - Gesta Normannorum Ducum des Wilhelm von Jumièges. Und zwar gehört unser Zusatz wie das ganze Buch VIII der Gesta zur heute so genannten Redaktion F, die Robert de Torigny um 1139 besorgte und die auch sonst Benoîts Hauptquelle ist. ${ }^{6}$

3 Der Fortgang der Erzählung zeigt, daß der Fremde nicht etwa zum Herzog zu kommen, sondern ihn zu erwarten versprach!

4 Andresen (1883: 340) vermißt in Benoîts Liste Wilhelm den Roten - aber der war doch nie Normannenherzog!

5 Andresen (1883: 338-340) zitiert nach Duchesne (1619: 315 D), nachlesbar auch bei Migne PL 149. 910C-911C. Moderne Ausgabe und Übersetzung: Van Houts (ed., 1992-1995: 2.282-287). 6 Van Houts (ed., 1992-1995): 1, S. LXXIX-XC. Das Originalms. dieser Redaktion ist erhalten, ibd. CIX f.; außerdem liegt sie in 22 Hss. vor, ibd. CXXVI ff. Durch ein bloßes Versehen erklärt 
Statt wie Benoît von neun kraftvoll regierenden Herzögen spricht Robert de Torigny von sieben Generationen, was auf dasselbe hinausläuft, da auf Richard III. sein Bruder Robert II., auf Robert Courteheuse sein Bruder Heinrich I. folgte. ${ }^{7}$ Was die dunkle Zeit nach der siebten Generation betrifft, so bemerkt Robert de Torigny zutreffend, «wir, die wir Heinrich I. überlebt haben», steckten jetzt in der Krise. ${ }^{8}$

Auch Robert de Torigny seinerseits folgt eng einer schriftlichen Quelle, der sog. Brevis relatio de Guillelmo Conquestore, die uns eindeutig schon aus der Zeit Heinrichs I. vorliegt, nämlich in einer Form aus den Jahren 1114-20 und einer ältesten Hs. aus den Jahren 1120-28. Zugesetzt hat Robert nur die Bemerkung, man lebe jetzt in der Krisenzeit nach der siebten Generation. ${ }^{9}$ Die Auszählung der sieben Generationen bis zu Heinrich I. gehört aber schon der Brevis Relatio an, auch wenn sie dort drei heutige Druckseiten vor unserer Erzählung erscheint. $^{10}$

Daß nun die Episode eine Erfindung der Zeit Heinrichs I. oder gar des Autors der Brevis Relatio selbst wäre, ist sehr unwahrscheinlich. Denn erstens erklärt der Autor einleitend, er habe die folgende Episode aus dem Munde vieler vernommen, die sie als wirklich geschehen bezeichneten. Und zweitens wird so

van Houts ibd. XCIV global, Benoît habe «die〉 Additamenta ausgelassen. Das stimmt gerade für unsere Episode nicht; das richtige Verhältnis hatte van Houts selbst (1984: 120-122) sehr klar dargestellt. $\mathrm{Zu}$ beanstanden ist dort nur, daß sie von einem knight from Brittany spricht who came to Rouen in the days of Henry I. Weder Benoît noch seine lat. Vorlage (noch deren sogleich zu besprechende Vorlage) lassen einen Zweifel daran, daß der Ritter nur zufällig an diesem Morgen von Rennes aufgebrochen ist und daß die Episode zur Zeit Rollos spielt, nach dem lat. Text eo tempore, quo primum pacificatus est cum rege Francie - also kurz nach 911, während Benoît die Episode, als er sie aus ihrer Nachtragsposition herausholte, in die RolloBiographie so einordnete, daß man glauben könnte, sie habe nach 922-23 stattgefunden (s. oben Anm. 1)

7 Irrig wiederum der Vorwurf von Andresen (1883: 340), Richard III. sei vergessen.

8 Von diesem (scheinbaren) Zählungsunterschied und dem zeitgerechten Lob Heinrichs II. abgesehen, hat Benoît seine Quelle nur in zwei Einzelheiten erweitert bzw. verändert: er hat hinzuerfunden, daß der Fremde an einem schönen Februarfesttag in Rouen ankam (was die Menge der Zuschauer plausibel macht), und er hat den Fremden in Avranches nach der dritten statt zur sechsten Tagesstunde speisen lassen (was den relativen Entfernungen Rennes-Avranches-Rouen besser gerecht wird). Insgesamt darf man das eine intelligente, zwar auf den königlichen Gönner schielende, aber in der Substanz treue Bearbeitung nennen - eine Charakterisierung, die Benoîts Chronik auch als Ganzes verdient.

9 Zum Verhältnis von Robert de Torignys Fassung der Gesta Normannorum Ducum zur Brevis Relatio vgl. van Houts (1984: 121 und ed. 1992-95, 1: LXXXV, XC f.); zu den Fassungen und Hss. der Brevis Relatio van Houts (1987: 164, 180-183). Der Text der Brevis Relatio bei Giles (1845: 1-23, unser Passus $17 \mathrm{f}$.).

10 Giles (1845: 14); Van Houts (ed. 1992-95: 1: LXXXV mit Anm. 246). 
leicht doch niemand die Regierungszeit des gegenwärtigen Herrschers als die wahrscheinlich letzte glückliche Epoche bezeichnen und damit das Unheil geradezu herbeireden. Wahrscheinlicher ist, daß sich die magische Zahl Sieben hier einnistete, als sie noch weit in die Zukunft wies. Doch auch wenn wir zur Frage der Entstehungszeit keine autoritative Antwort zu bieten haben, lohnt es, die Episode zu analysieren.

Die Hauptgestalt reitet auf einer bewegten Wasserfläche, ohne sie zu berühren, und legt bequem Tagereisen zurück, die menschliches Vermögen um ein Mehrfaches übersteigen; sie bleibt anonym selbst gegenüber ihrem freiwilligen Gastgeber, wird von diesem aber ohne weiteres - und wie sich zeigt, zu Recht für fähig gehalten, bis in die ferne Zukunft zu blicken; sie diktiert in Mißachtung der menschlichen Hierarchie dem Herzog enge Bedingungen für ein mögliches Treffen und setzt beim ersten Tageslicht eine Fahrt fort, von der in diesem Erzählhorizont nie der Anfangs- noch der Endpunkt noch der Sinn zu erfahren sein werden. Gerade weil hier verschiedene, voneinander logisch unabhängige übernatürliche Fähigkeiten zusammenkommen, handelt es sich sichtlich obwohl der Reiter auf Fragen nach seiner Identität sich selbst kryptisch als «einen Menschen` bezeichnet - um eine übernatürliche Gestalt, die nur «auf der Durchreise> durch die Normandie kommt.

Da die Autoren der erhaltenen Fassungen Christen sind, müssen wir uns zunächst bemühen, die Episode in den Kategorien christlicher Übernatürlichkeit zu verstehen. Aber gerade das will nicht gelingen. Daß der Reiter kein böser Geist ist, schließen schon die Zeitgenossen daraus, daß er ihnen über seinen Ritt von Avranches aus die Wahrheit gesagt hat, Spätere dann daraus, daß seine Prophezeiungen eintreffen. Aber ebenso wenig gelingt es, in ihm einen Engel zu sehen. «Normalerweise` kommen Engel vom Himmel herab und kehren dorthin zurück, erscheinen und verschwinden wohl auch übergangslos. Sie können auch vorgeben, auf Reisen zu sein wie die drei Engel von Abraham, oder sie können einen Reisenden schützend begleiten wie Raphael den Tobias. Doch unser Reiter vollführt einsam seine Gewaltritte, die sein Dauerschicksal zu sein scheinen.

So werden wir auf heidnische Vorstellungen und damit im wesentlichen auf die altnordische Überlieferung verwiesen. Dort ziehen einige Erzählungen schon durch die Gesamtstruktur unsere Aufmerksamkeit auf sich. Daß sie in der uns vorliegenden Form erst aus der Zeit zwischen etwa 1200 und kurz nach 1300 stammen, erklärt sich zum Teil schon aus den Überlieferungsverhältnissen altnordischer Prosa überhaupt und sagt über die Entstehungszeit zumindest ihrer Vorstufen und des Erzähltyps noch nichts aus.

Um 1208 soll bei einem Schmied in Nesjar ein Reiter um Nachtquartier gebeten haben. Während er am nächsten Morgen sehr früh sein Pferd beschlagen 
läßt, erzählt er, die Nacht vor seinem Hierherkommen habe er in Medaldal in Telemark zugebracht, die kommende werde er in Sparmork auf dem Weg nach Schweden verbringen. Der Schmied hält beides für unmöglich; Sparmork liegt sieben Tagesritte entfernt. Der Fremde hat den Schmied schon einen unverständigen und unweisen Mann genannt, weil er nichts frage. Jetzt nennt sich Odin mit Namen, setzt mit dem Pferd aus dem Stand über einen hohen Zaun und ist verschwunden. Kurz darauf findet in Schweden die Schlacht von Lena statt. ${ }^{11}$

Hier ist also der Zweck seiner ruhelosen Ritte noch erkennbar: er eilt auch jetzt noch von Schlacht zu Schlacht, offenbar doch, um in sie einzugreifen. Insofern bleibt ihm in dieser Erzählung noch viel von seiner alten Funktion. Wenn demgegenüber unsere normannische Erzählung seinen Ritten nicht etwa eine andere, sondern gar keine Funktion zuweist, so muß sich die Frage melden, ob nicht in der früher christianisierten Normandie diese Erzähllücke einfach auf einem opportunen Verschweigen der einst identischen Funktion beruht - wobei ein Erzähler zunächst sogar darauf rechnen konnte, daß die Zuhörerschaft dies nicht als Informationslücke, sondern als beredtes Verschweigen - sapienti sat! - verstand. Selbst in den «klassischen〉 Zeugnissen der altnordischen Religion trat Odin ja meist anonym oder pseudonym auf; gab er sich indirekt zu erkennen (wie in der Vafthrúđnismál und den Heiđreksrätseln) oder nannte er sich gar namentlich (wie in der Grímnismál), so bedeutete dies für seinen nichtgöttlichen Gesprächspartner den Untergang.

Da Rollo erst als Erwachsener Christ wurde, sind für uns auch jene vier Erzählungen von Interesse, bei denen Odin an die beiden Norwegerkönige herantritt, die in derselben Lage sind: je zwei Erzählungen über Olaf Tryggvason und Olaf den Heiligen. Sie sind, wie zu erwarten, prochristlich tendenziös, aber in verschiedenem Grade. ${ }^{12}$

Olaf Tryggvason erhält eines Abends Besuch von einem Einäugigen, der ihn großartig bis in die späte Nacht mit seiner unerschöpflichen Kenntnis fremder Länder und alter Zeiten unterhält. Am nächsten Morgen ist der Fremde nicht mehr zu finden, hat aber den Köchen Fleischbrocken für das Mahl des Königs übergeben. Daran erkennt der König nun Odin und läßt das Fleisch wegschaffen. ${ }^{13}$

Aggressiver reagiert Olaf der Heilige. Ein Fremder mit dem Decknamen Gest (〈Gast〉) erzählt dem König abends viel von den Vorzeitkönigen. An einer Frage,

11 Hákonarsaga Sverrissonar Kap. 20.

12 Zusammenhängend behandelt sie z. B. Golther (1908: 342-44).

13 Snorri Sturluson, Óláfssaga Tryggvasonar Kap. 64 (dt.: Thule 14.271f.); Óláfssaga Tryggvasonar hin mesta Kap. 198. 
die Olafs Christenglauben in Versuchung bringen könnte, erkennt der König Odin und schlägt nach ihm mit dem Meßbuch; Odin verschwindet. ${ }^{14}$

Auch die beiden verbleibenden Erzählungen werden üblicherweise als Odinerzählungen verstanden. Hier kommt Odin unter den Decknamen Nornagest («Nornengast`) bzw. Tóki zu Olaf Tryggvason bzw. Olaf dem Heiligen, erzählt auch hier abends spannend aus uralten Heldenzeiten, an denen er selbst teilgenommen zu haben erklärt, empfängt dann aber auf eigene Bitte die Taufe und stirbt als Christ. ${ }^{15}$

Die beiden letztgenannten Erzählungen wollen also den Heidengott in Frieden, doch unwiderruflich begraben; die beiden ersten lassen ihn als verführerischen Dämon weiterleben. In allen vier Erzählungen aber bewährt sich der erst im Erwachsenenalter getaufte König gegenüber dem unheimlichen abendlichen Geschichtenerzähler als Christ. «So verschieden die Berichte im einzelnen auch sind, die Grundlage bleibt überall dieselbe» (Golther 1908: 342).

Lenken wir von hier aus den Blick auf Rollo zurück, so wird klar, daß bei ihm dieselbe Erzähltendenz wirkt. Jeden Gedanken an eine Sympathie oder gar Kollusion des Herzogs mit dem dubiosen und im Christenglauben nicht unterzubringenden Fremden macht der Erzähler von vornherein unmöglich, indem Rollo gar nicht mit ihm zusammentrifft, ja als einziger ihn als böses phantasma verdächtigt. Der Christenglaube des Neuchristen soll auch hier außer allem Zweifel stehen. Man kann es deshalb schwerlich für Zufall halten, wenn sich auch die normannische Erzählung gerade an den konvertierten Herrscher heftet und nicht an eine andere Generation. Die Konversion ist das Ereignis, das Erzähler und Publikum noch einmal und vielleicht zum letzten Mal magisch an den 〈Fremden〉 denken läßt, und der Konvertit bzw. in unserem Fall seine ganze Dynastie muß gestärkt daraus hervorgehen.

Aber passen nun die sonstigen Eigenschaften des Reiters zu Odin? Durchaus:

- $\quad$ Er reitet über die Wellen. Über das Meer ritt auch jener geheimnisvolle Einäugige hinweg, der sich des jungen vaterlos umherirrenden Helden Hadding angenommen, ihn mit einem Zaubertrank gestärkt und ihm ein Stück Zukunft verkündet hatte - eine eindeutige Odinserscheinung. ${ }^{16}$

- Unser Reiter ist ein stattlicher Mann: Bel home $i$ out a grant maniere / De cors, de façon et de chere. Und von Odin sagt Snorri: ${ }^{17}$ «er war so schön und

14 Óláfssaga helga, Anhang in den Fornmanna sögur $5.171 \mathrm{f}$.

15 Nornagests Tháttr (dt.: Thule 21.199-218); Tháttr Tóka Tókasonar (Fornmanna sögur 5.299303).

16 Saxo Grammaticus, Gesta Danorum I 6 (Holder S. 24, dt. Herrmann 1901: 28 f.).

17 Snorri Sturluson, Ynglingasaga Kap. 6f., auch zum Folgenden (dt.: Thule 14.31 ff.); vgl. auch Golther (1908: 309f.). 
vornehm anzuschauen, wenn er bei seinen Freunden saß, daß allen das Herz darüber lachte; aber wenn er im Heer war, da schien er seinen Feinden grimmig».

- Unser Reiter gewinnt das Vertrauen aller, die mit ihm zusammentreffen. Und Odin, wieder nach Snorri, besaß «die Kunst, daß er beredt und glatt sprach, daß das allein allen, die es hörten, wahr däuchte».

- Vor allem: Unser Reiter blickt in die Zukunft. Das konnte natürlich auch der «Gott der Weisheit»: Durch seine Zauberkunst vermochte er, um abermals Snorri zu zitieren, «die Geschicke der Leute und ungeschehene Dinge zu wissen».

Man kann es nicht leugnen: unsere ganze Erzählung kommt einer Odinserzählung so nahe, daß von Zufall keine Rede sein kann. Damit ist freilich noch nicht entschieden, ob einst der erste Erzähler

1. Odin namentlich nannte oder

2. ihn anonym beließ, aber erkannt wissen wollte oder schließlich ob er

3. unter Benutzung eines schon in der De-Wodanisierung befindlichen Erzählschemas ${ }^{18}$ bona fide einfach von einem geheimnisvollen Fremden sprach.

Die erste dieser Annahmen hätte reale Chancen nur, wenn wir den Mut hätten, die Erzählung wirklich in die Zeit Rollos zu datieren. Rollo kommt nach Frankreich als Führer einer Gefolgschaft, deren Bindung prinzipiell nicht erblich ist; er sieht bei seinen neuen Pairs faktische Erblichkeit und will nun auch eine Dynastie gründen. Die Haupttradition der Normannenhistoriker wird ihn als ehrlichen Christen bezeichnen; aber wenige Jahrzehnte nach seinem Tod behauptet eine Fassung des um 942 entstandenen Planctus auf seinen frommen Sohn Wilhelm Langschwert, Rollo sei als Heide gestorben, ${ }^{19}$ und ein knappes Jahrhundert später erklärt Adémar von Chabannes gar, Rollo habe auch nach der Taufe christliche Gefangene den Heidengöttern geopfert ${ }^{20}$ - nach unserem Wissen über die altgermanische Religion hieße das essentiell: dem Odin. Stimmt wenigstens die Grundtendenz beider Nachrichten, so kann Rollo gerade an die Adresse seiner alten heidnischen und schwer zum erblichen Gehorsam bereiten

18 Nach einer auch heute wohlvertretbaren Forschungsmeinung beruhen ja die zahllosen Sagen vom Wilden Jäger und von seinen regionalen Ersatzgestalten in fast dem ganzen germanischen Sprachgebiet letztlich auf einer De-Wodanisierung, ohne daß die «normalen` Erzähler und Hörer das noch merken.

19 Vgl. Douglas (1942: 433).

20 Historia III 20. Die längere Fassung C präzisiert, er habe beim Nahen seines Todes den Heidengöttern hundert christliche Gefangene, dem Christengott hundert Pfund Gold geopfert. 
Mitkämpfer eine Prophezeiung im wirksamsten Namen, nämlich Odins, in Umlauf gesetzt haben nach dem Motto: Die Gottheit selbst verlangt von euch Gehorsam gegenüber dem neuen Herzogsgeschlecht.

Von Wilhelm Langschwert an sind die Herzöge unbezweifelte Christen: eine offene Odinsprophezeiung zugunsten ihrer selbst könnten sie nicht mehr ohne Skandal in Umlauf setzen, und ihre noch heidnischen Gegner täten es aus Interessegründen erst recht nicht. Aber in der Normandie koexistieren weiterhin zwei Religionen. In Lisieux und Avranches scheinen die Bischofsstühle bis 990 leerzustehen, fünf Bischöfe des westnormannischen Bistums Coutances müssen überhaupt im ostnormannischen Rouen residieren. ${ }^{21}$ Der große westnormannische Aufstand des Riulf von 933/35 gegen Wilhelm Langschwert ist ein skandinavischer und damit essentiell ein heidnischer Aufstand; er ist zunächst erfolgreich bis unter die Mauern von Rouen. Auch auf Wilhelms Ermordung folgt klar eine heidnische Reaktion. Doch sogar die Herzöge selbst verschmähen im Kampf gegen Zentralfranzosen nach 942 und 965 nicht die Hilfe von Flotten aus dem heidnischen Skandinavien; erst die dritte und letzte solche Aktion 1014-15 steht unter Olaf, dem späteren Heiligen. Noch 1047 droht in der Schlacht von Val-ès-Dunes die Normandie im Kampf zwischen Westnormannen und Ostnormannen (diese jetzt verbündet mit Zentralfranzosen) zu zerbrechen, ${ }^{22}$ und noch bis 1066 konzentrieren sich die normannischen Klöster mit Ausnahme des Mont-Saint-Michel in frappantem Maße auf die Ost- und Mittelnormandie. ${ }^{23}$ In der Westnormandie fehlt damit großenteils noch jene mönchische Aktivität, die zunächst einmal den Adel auch innerlich an das Christentum heranführt. Was selbst in der Oberschicht noch möglich ist, zeigt eine Anekdote. Der Erzbischof Mauger von Rouen, ein Sohn Richards II. und damit Onkel Herzog Wilhelms, des künftigen Eroberers, zog sich nach seiner Absetzung 1054 in die Westnormandie einschließlich der Kanalinseln zurück. Noch mindestens ein halbes Jahrhundert später erzählte man sich dort, Mauger habe ständig Gespräche geführt mit un deiable [...] privé namens Toret; ${ }^{24}$ nach Abzug der romanischen Hypokoristikon-Endung -et ist das der Gottesname Thor. Wenn nun heidnisches Glaubensgut, jetzt als Aberglaubensgut, selbst bei einem in der Ostnormandie

21 Douglas (1942: 433).

22 Gegenstandslos ist allerdings die immer mal wieder (z. B. bei Léonard 1948: 38) auftauchende Behauptung, in dieser Schlacht hätten die Heiden noch Thor aïe! gerufen; sie beruht auf einer Manipulation am Text des Roman de Rou.

23 Douglas (1995: 120 f.)

24 Roman de Rou III 4577, 4578, 4580. Wace selbst wurde um 1110 auf der Kanalinsel Jersey geboren und hat zweifellos dort in seiner Jugend diese Lokaltradition gehört (Holden ed. 197073, Bd. III 15 und 164). 
aufgewachsenen Mitglied der Oberschicht durch ein Zufallszeugnis noch so spät für uns dokumentierbar ist - wie viel stärker und länger mag es im Verborgenen speziell bei den Unterschichten und speziell im Westen weitergeblüht haben? Alles das macht dann auch die obengenannte dritte Möglichkeit sehr unwahrscheinlich: man kann nicht gut - spätestens gegen 1100 - in der Normandie unsere alles andere als naiv-einfache Erzählung erfinden, ohne überhaupt $\mathrm{zu}$ merken, welcher heidnisch abergläubischen Interpretation sie fähig ist. Und eine Interpretation, die ein Autor voraussieht und nicht abblockt, sondern zumindest billigend in Kauf nimmt, ist eine legitime Interpretation.

Damit optieren wir also für die zweite Interpretationsmöglichkeit. Unsere Erzählung, Tendenzerzählung eines Christen zugunsten des Herzogshauses, richtet sich von vornherein auf die breite Grauzone im damaligen durchschnittlichen normannischen Bewußtsein zwischen christlichen und heidnischen Vorstellungen. Die Erzählung ist ambivalent genug, um einerseits christlichem Denken gerade noch kein Ärgernis zu geben, andererseits heidnisches Denken bzw. dessen zum Aberglauben gewordene Reste erschauern zu lassen bei der Frage, wer sich hinter dem 〈Fremden〉 verbirgt - und je größer dieser Schatten ist, desto schicksalsschwangerer natürlich auch seine Prophezeiung. Insgesamt wirkt die Erzählung also gerade durch ihre Ambivalenz integrierend.

Was das Entstehungsdatum angeht, neigen wir zu einer gemäßigten Frühdatierung, damit in der Zahl von sieben Generationen zunächst noch eine Verheißung steckt und nicht ein Countdown. Robert de Torigny trägt die Prophezeiung dann durch ihre eigene und der Normandie Krise, Benoît schließlich hat das Glück und das Darstellungsgeschick, sie, die jetzt zur Gänze Geschichte geworden ist, mit neuem Glanz auszustatten.

\section{Bibliographie}

Andresen, Hugo, 1883/1886: «Über die von Benoît in seiner normannischen Chronik benutzten Quellen, insbesondere sein Verhältnis zu Dudo, Wilhelm von Jumièges und Wace». In: Romanische Forschungen 1.327-412, 2. 477-538.

Benoît: s. Fahlin.

Brevis Relatio: s. Giles.

Douglas, David C., 1942: «Rollo of Normandy». In: The English Historical Review 228.417-36. Douglas, David C., 1995: Wilhelm der Eroberer, Herzog der Normandie. [Dt. Übs. von William the Conqueror, London.] 'München.

Duchesne, André, ed., 1619: Historiae Northmannorum Scriptores antiqui. Paris.

Egilsson, Sveinbjörn [u. a.], edd., 1825-37: Fornmanna sögur eptir gömlum handritum, 12 Bde., Kopenhagen.

Fahlin, Carin, ed., 1951-54: Chronique des Ducs de Normandie par Benoît, Bd. I, II, Uppsala. [S. auch Södergård.] 
Fornmanna sögur, s. Egilsson [u. a.].

Giles, John Allen, ed., 1845: Scriptores rerum gestarum Wilhelmi Conquestoris, London

[Nachdruck New York (Franklin) 1967].

Golther, Wolfgang, 1908: Handbuch der germanischen Mythologie. '2Leipzig.

Hákonarsaga Sverrissonar, z. B. in: Konunga Sögur, ed. Gudni Jónsson, Reykjavik 1957.

Herrmann, Paul, 1901: Erläuterungen zu den ersten neun Büchern der Dänischen Geschichte des Saxo Grammaticus, I, Übersetzung, Leipzig.

Holden, A. J., ed. 1970-73: Le Roman de Rou de Wace. 3 Bde., Paris (Picard, Société des Anciens Textes Français).

Léonard, Émile-G., 1948: Histoire de la Normandie. Paris (PUF; Que sais-je 127).

Migne, J.-P.: Patrologiae cursus completus [...] Series secunda in qua prodeunt patres [...] ecclesiae latinae, 221 Bde,. Paris 1844-64.

Olrik, Jörgen und Raeder, Hans, edd., 1931: Saxonis Gesta Danorum [...] I (Text), Kopenhagen.

Saxo Grammaticus: s. Olrik/Raeder.

Nornagests Tháttr, Teil von [Bergr Sokkason?] Ólafssaga Tryggvasonar hin mesta; s. diese. Óláfssaga Tryggvasonar hin mesta, ed. Ólafur Halldórsson, 3 Bde., Kopenhagen 1958 ff. (Editiones Arnamagnaeanae A1-3).

Snorri Sturluson, Heimskringla, ed. Bjarni Adalbjarnarson, 3 Bde., Reykjavik 1941-51 (Íslenzk fornrit).

Snorri Sturluson, Óláfssaga Tryggvasonar, Teil der Heimskringla; s. diese.

Snorri Sturluson, Ynglingasaga, Teil der Heimskringla; s. diese.

Södergård, Östen, 1967: wie Fahlin 1951-54, Bd. III, Glossaire. Lund.

Tháttr Tóka Tókasonar, in: Fornmanna sögur 5.299 ff.; s. Egilsson [u. a.].

Thule. Altnordische Dichtung und Prosa. 24 Bde. und Einleitungsband, Jena 1912-30.

[Nachdruck mit neuen Nachworten Darmstadt 1963-67.]

Van Houts, Elisabeth, 1984: "The adaptation of the Gesta Normannorum Ducum by Wace und Benoît». In: Non Nova, sed Nove. Mélanges de civilisation médiévale dédiés à Willem Noomen, ed. Martin Gosman und Jan van Os, Groningen 1984, 115-124.

Van Houts, Elisabeth, 1987: «The ship list of William the Conqueror». In: Anglo-Norman Studies (Proceedings of the Battle Conference) 10.159-183.

Van Houts, Elisabeth, ed., 1992-95: The Gesta Normannorum Ducum of William of Jumièges, Orderic Vitalis, and Robert of Torigni, Oxford.

Wace: s. Holden.

Wilhelm von Jumièges: s. Van Houts, ed., 1992-95. 